Sādhanā Vol. 37, Part 1, February 2012, pp. 107-124. (C) Indian Academy of Sciences

\title{
Fatigue crack growth due to overloads in plain concrete using scaling laws
}

\author{
SONALISA RAY ${ }^{\mathrm{a}}$ and J M CHANDRA KISHEN ${ }^{\mathrm{a}, *}$ \\ ${ }^{a}$ Department of Civil Engineering, Indian Institute of Science, \\ Bangalore 560012 , India \\ e-mail: chandrak@civil.iisc.ernet.in
}

\begin{abstract}
Scaling laws are represented in power law form and can be utilized to extract the characteristic properties of a new phenomenon with the help of self-similar solutions. In this work, an attempt has been made to propose a scaling law analytically, for plain concrete when subjected to variable amplitude loading. Due to the application of overload on concrete structures, acceleration in the crack growth process takes place. A closed form expression has been developed to capture the acceleration in crack growth rate in conjunction with the principles of dimensional analysis and self-similarity. The proposed model accounts for parameters such as, the tensile strength, fracture toughness, overload effect and the structural size. Knowing the governed and the governing parameters of the physical problem and by using the concepts of self-similarity, a relationship is obtained between the different parameters involved. The predicted results are compared with experimental crack growth data for variable amplitude loading and are found to capture the overload effect with sufficient accuracy. Through a sensitivity analysis, fracture toughness is found to be the most dominant parameter in accelerating the crack length due to application of overload.
\end{abstract}

Keywords. Fatigue; variable amplitude; plain concrete; dimensional analysis; self-similarity; size-effect.

\section{Introduction}

Scaling laws are widely used in science as well as in engineering and provide a very important information about the phenomenon under consideration. It gives an evidence of the property self-similarity, wherein, the phenomenon under consideration reproduces itself under different time and space scales. By making use of these self-similar solutions, researchers have attempted to predict the properties of the new phenomena. By constituting self-similar solutions, computations as well as the description of various parameters, influencing the phenomenon becomes simpler. Further, most importantly, it could be employed to solve a complex phenomenon in an approximate way. Hence, scaling laws have special and unique importance. One of the widely used scaling laws to study the fatigue crack propagation, is the Paris law which is found to exhibit a weak form of scaling (Ciavarella et al 2008).

*For correspondence 
Structures such as airport pavements, highways, railway bridges, etc. are subjected to repetitive loading during their design life. The amplitude of cyclic loading rarely remains constant during the service period. Generally, these structures are subjected to wide spectrum of load amplitude. The crack growth behaviour alters with the sudden insertion of higher amplitude load cycles within a constant amplitude history (known as overloads). A schematic representation of the overload phenomenon with constant amplitude loading has been shown in figure 1. Unlike metals, deceleration in crack growth does not occur in concrete due to sudden overloads. Concrete like quasi-brittle materials when subjected to repeated loads with different amplitude, exhibits complicated behaviour due to the presence of large size process zone ahead of the crack tip. Further, the sequence by which the load is being applied plays a very important role. Hilsdorf \& Kesler (1964) observed that the fatigue strength of concrete increases due to the application of few numbers of high amplitude loading, when the loading system shifts from higher amplitude loading to lower. On similar lines, Holmen (1979), Oh (1993), Cornelissen \& Reinhardt (1984) have attempted to understand the sequence effect by conducting experiments. Very few attempts have been made to predict the crack growth analytically in plain concrete when subjected to variable amplitude fatigue loading. Slowik et al (1996) have proposed an empirical law to study the crack propagation, based on linear elastic fracture mechanics (LEFM) concept. They have considered a loading system interrupted by spikes. This law applicable to variable amplitude fatigue loading is described by

$$
\frac{d a}{d N}=C \frac{K_{I \max }^{m} \Delta K_{I}^{n}}{\left(K_{I C}-K_{I \text { sup }}\right)^{p}}+F(a, \Delta \sigma),
$$

where $K_{\text {Isup }}$ is the maximum stress intensity factor ever reached by the structure in its loading history and $F(a, \Delta \sigma)$ is a function which takes into account the effect of overloads. The coefficients $m, n$ and $p$ are constants for all structural sizes and are determined through an optimization process. To include the effect of overload in the empirical model, they have defined an overload function $F$ which is dependent on two parameters, change in stress due to application of overload and crack length. No closed form expression has been provided by the authors to predict

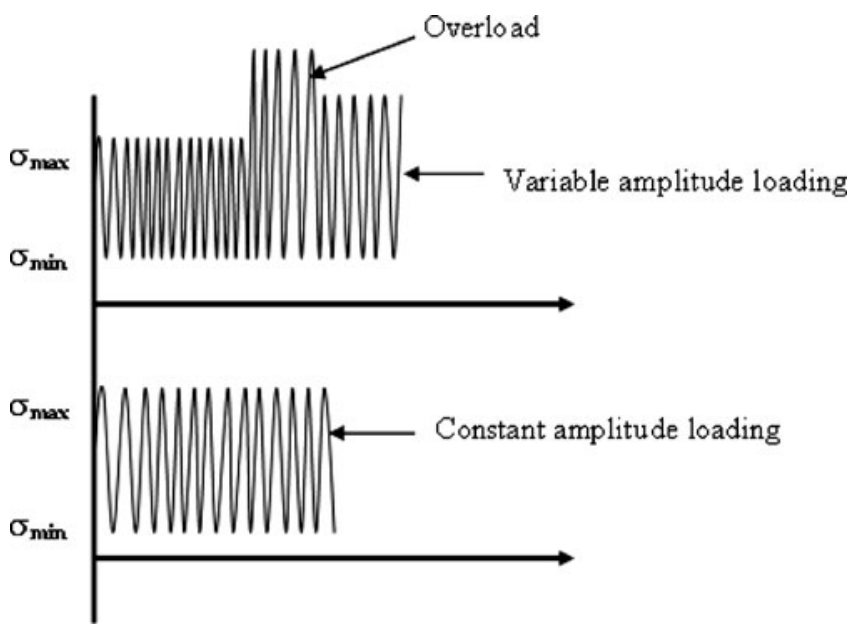

Figure 1. Representation of constant and variable amplitude loading. 
increase in crack growth. In concrete, damage due to cyclic loading is observed to occur primarily in the fracture process zone (FPZ) Hordijk \& Reinhardt (1989). If the FPZ shows greater sensitivity towards cyclic loading than the surrounding material, then the fatigue bahaviour is said to be dependent on loading history (Slowik et al 1996). Slowik et al (1996), in their experimental study have observed the crack growth rate to accelerate when the structure is reloaded with a fully developed FPZ. Hence, spikes in the loading history accelerates the crack propagation in concrete unlike metals wherein, a deceleration in crack growth takes place. To capture the increase in crack propagation due to presence of overloads, Sain \& Chandra Kishen (2007) have proposed an empirical formulation.

Most of the fatigue crack propagation laws available in the literature are purely empirical in nature and are not dimensionally consistent. Further, the existing laws are a modified form of Paris law wherein, linear elastic fracture parameters are used. But concrete exhibits nonlinear behaviour due to the presence of large size process zone ahead of the crack tip and hence this nonlinearity which influences the crack propagation must be considered in the analytical formulation. In spite of the fact that the structural components experience repetitive loading of variable magnitude, most of the analytical models have been developed for cyclic loading of constant magnitude. In this study, a crack propagation model is proposed for plain concrete which can capture the sudden increase in crack length when subjected to overloads.

\section{Fatigue behaviour in plain concrete}

In the case of metals and ceramics, fatigue fracture has been studied extensively but for concrete, however, the knowledge of fatigue fracture is limited. This is due to the fact that fracture behaviour in concrete is more complicated because of its heterogeneous nature and presence of large size fracture process zone (FPZ) at the crack tip. To understand the fatigue behaviour of concrete, it is essential to understand the existing literatures related to fatigue of plain concrete based on fracture mechanics concept. The crack propagation study under fatigue loading began with the well-known Paris law which is found to exhibit a weak form of scaling. The earlier assumption about the Paris law coefficients as material constants has been proved to be invalid by various researchers (Spagnoli 2004; Carpinteri \& Paggi 2007; Ciavarella et al 2008; Ray \& Chandra Kishen 2011). In spite of the fact that the linear elastic assumption in Paris law makes it inapplicable for quasi-brittle materials like concrete, attempts have been made by many researchers to apply Paris law for the study of fatigue behaviour in concrete. One of the earliest attempts is by Bazant \& Xu (1991) who have introduced the size effect into the Paris law using the size dependent fracture toughness $\left(K_{I C}\right)$. Recently, various authors have investigated the fatigue behaviour of concrete by adopting dimensional analysis and self-similarity approach. On this aspect, Spagnoli (2004), Carpinteri et al (2009), Ciavarella et al (2008), Carpinteri \& Paggi (2007, 2009), and Paggi (2009) came up with more generalized form of Paris law by considering many crack growth influencing parameters. Carpinteri \& Spagnoli (2004) have proposed a size dependent crack growth law using the fractal geometry concept together with the idea of self-similarity. Spagnoli (2004) has derived a crack size dependent Paris law based on the assumption of incomplete self-similarity in the crack propagation model. In a study by Carpinteri \& Paggi (2007), the existence of correlation between the Paris law coefficients $C$ and $m$ has been illustrated. They have applied the concept of dimensional analysis to the linear range of fatigue crack growth. Ciavarella et al (2008) have generalized the Barenblatt dimensional analysis approach (Barenblatt 1996, 2004) to study the functional dependencies of $m$ and $C$ on additional dimensionless parameters than the ones used in the original Barenblatt model using 
experimental data for both steel and concrete. The developed correlation between $C$ and $m$ is reported to be different for steel and concrete. On similar lines, Carpinteri \& Paggi (2009) and Paggi (2009) have constructed a generalized Wohler's and Paris equations analytically. It is observed that the parameters, micro-structural size and crack size in both the relationships are size-scale dependent. In the recent study by Ray \& Chandra Kishen (2011), an improved law has been proposed to predict the crack propagation in the linear range of crack growth due to fatigue loading by considering nonlinear fracture parameter which is more appropriate for concrete-like material. The proposed model is able to capture size effect appropriately which is an important aspect in fracture mechanics study of concrete structures. Further, in another study by the same authors, Ray \& Chandra Kishen (2010), they have employed the population growth model to study the fatigue crack propagation in plain concrete. An improvement over the previous model has been seen due to the applicability of the model in both zone II and zone of III of the Paris regime. The above discussed fatigue crack growth models can be used to predict the crack growth due to the application of constant amplitude loading which occur rarely in real loading scenario. Moreover, energy-based parameters need to be taken into account to analyse the fatigue crack growth of concrete as these are more appropriate for quasi-brittle materials. In this work, an attempt has been made to propose a fatigue law for studying the crack propagation when the structure is loaded with variable amplitude cyclic loading. Moreover, the sudden increase in crack growth rate due to overloads can be predicted using this proposed law.

\section{Research significance}

A fatigue crack propagation law has been proposed for plain concrete, to understand the response of the structure when subjected to varying load amplitude. Due to the application of overload on concrete structures, acceleration in the crack growth process takes place. In this study, a closed form expression has been developed to capture the acceleration in crack growth rate in conjunction with the principles of dimensional analysis and self-similarity. The proposed model includes the crack growth influencing parameters such as fracture toughness, crack length, structural size and changes in the fatigue load amplitude. To verify the accuracy of the developed model, a validation study has been carried out with the available experimental data in the literature.

\section{Scaling laws and dimensional analysis}

Scaling laws or power-laws describe the power-law relationship between different quantities. These are not just an approximation to the experimental data, rather they reveal a very important property of the considered phenomenon known as self-similarity. The self-similar solutions can be utilized to acquire the properties of the phenomenon under consideration at different time and space scale through similarity transformation (Barenblatt 1996). One of the approachs to bring out the information is through scaling laws. In construction of an analytical model, it is impossible to take into account all the factors which influence the phenomenon. So, every model is based on certain idealization of the phenomenon. In constructing these idealizations, the phenomenon under study should be considered at intermediate times and distances. Therefore, every mathematical model is based on intermediate asymptotic. Infact, self-similar solutions are always intermediate asymptotic to the solution of broader class of problem (Barenblatt 1996). 
Intermediate asymptotic behaviour means the behaviour in the regions where, these solutions have ceased to depend on the details of the initial conditions and boundary conditions (Barenblatt 2004). In other words, the intermediate asymptotic is a time-space dependent solution of an evolution equation which has already forgotten its initial conditions, but still does not feel the limitations imposed by the system boundary. Figure 2 shows a typical demonstration of intermediate asymptotic where, surface waves are generated by vibration of triangular element on surface of originally still liquid confined in a rectangular container (top view). As the waves propagate outwards, they gradually obtain the natural circular shape, being undisturbed by either boundary. This happens at the intermediate distance between the container center and the container wall. The solution describing the circles is an intermediate asymptotic (IA) of the system (Ruzicka 2008). Figure 3 represents all the phenomena in the universe through nesting of similarity concepts.

According to dimensional analysis, any physical problem can be written in the form

$$
a=f\left(a_{1}, \ldots \ldots, a_{k}, b_{1}, \ldots ., b_{n}\right) .
$$

Here, $a$ is the quantity to be determined in the phenomenon and is found to be dependent on the arguments on the right hand side. In Equation 2, it is assumed that the the parameters $b_{1}, \ldots \ldots, b_{n}$ have independent physical dimensions. Independent physical dimension means the governing arguments $a_{1}, \ldots \ldots, a_{k}$ as well as governed quantity $a$ can be expressed in terms of $b_{1}, \ldots . ., b_{n}$. Equation 2 can be expressed as

$$
\Pi=\Phi\left(\Pi_{1}, \ldots . ., \Pi_{k}\right),
$$

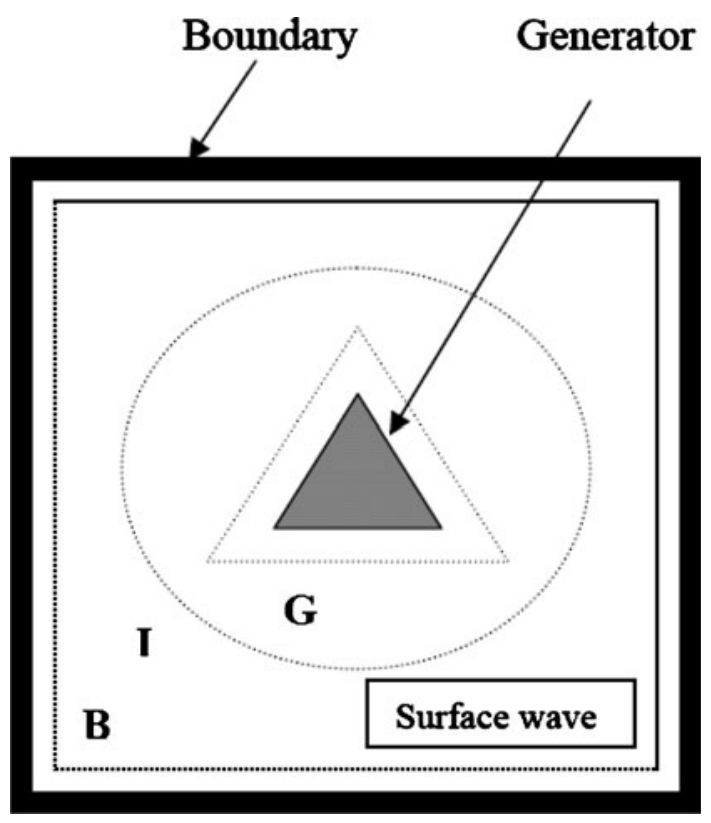

Figure 2. Demonstration of intermediate asymptotic. 


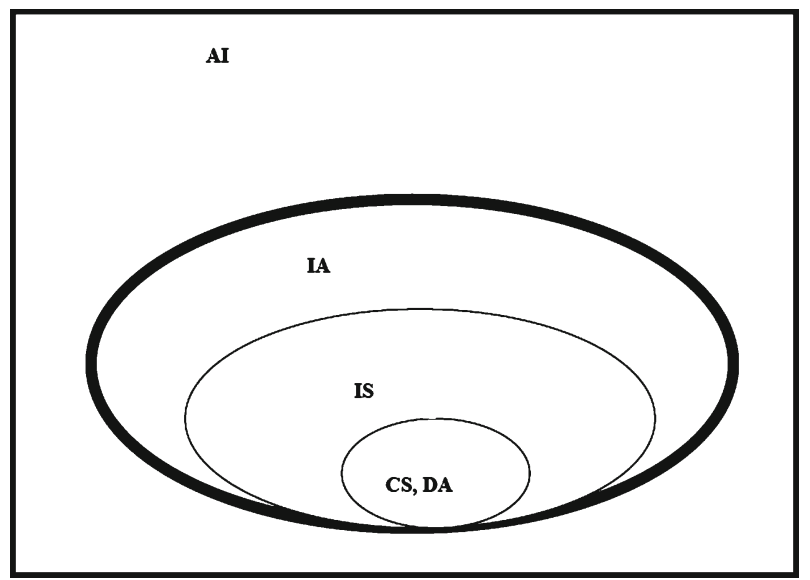

Figure 3. Nesting of similarity concepts. DA: dimensional analysis; CS: complete similarity; IS: incomplete self- similarity; IA: intermediate asymptotic; AI: absence of intermediateness.

where the dimensionless parameters are defined by the relation

$$
\begin{gathered}
\Pi=\frac{a}{a_{1}^{p}, \ldots \ldots, a_{k}^{r}}, \quad \Pi_{1}=\frac{a_{1}}{a_{1}^{p 1}, \ldots \ldots, a_{k}^{r 1}}, \quad \ldots \ldots . . \quad \Pi_{k}=\frac{a_{k}}{a_{1}^{p k}, \ldots \ldots, a_{k}^{r k}} \\
a=a_{1}^{p}, \ldots \ldots ., a_{k}^{r} \Phi\left(\frac{a_{1}}{a_{1}^{p_{1}} \ldots \ldots a_{k}^{r_{k}}}, \ldots ., \frac{a_{n}}{a_{1}^{p_{n}} \ldots \ldots a_{k}^{r_{k}}}\right) .
\end{gathered}
$$

Let us consider the parameter $a_{1}$. This parameter is considered as non-essential if the corresponding dimensionless parameter $\Pi_{1}$ is too large or too small (tend to zero or infinity), giving rise to a finite non-zero value of the function $\Phi$ with the other similarity parameters remaining constant. The number of arguments can now be reduced by one and we can write Equation 3 as

$$
\Pi=\Phi_{1}\left(\Pi_{2}, \ldots ., \Pi_{k}\right),
$$

where, $\Phi_{1}$ is the limit of the function $\Phi$ as $\Pi_{1} \rightarrow 0$ or $\Pi_{1} \rightarrow \infty$. This is called complete selfsimilarity or self-similarity of the first kind (Barenblatt 1996). On the other hand, for $\Pi_{1} \rightarrow 0$ or $\Pi_{1} \rightarrow \infty$, if $\Phi$ also tends to zero or infinity, then the quantity $\Pi_{1}$ becomes essential, no matter how large or small it becomes. However, in some exceptional cases, the limit of the function $\Phi$ tends to zero or infinity, but the function $\Phi$ has power type (scaling) asymptotic representation which can be written as

$$
\Phi \cong \Pi_{1}^{\gamma} \Phi_{1}\left(\Pi_{2}, \ldots ., \Pi_{k}\right),
$$

where the constant $\gamma$ and the non-dimensional parameter $\Phi_{1}$ cannot be obtained from the dimensional analysis alone. This is the case of incomplete self-similarity or self-similarity of the second kind. It can be noted here that, the parameter $\gamma$ can only be obtained either from a best fitting procedure on experimental results or according to numerical simulations. In such cases, self-similar solutions are obtained by solving the nonlinear eigen value problem. Sometimes for $\Pi_{1} \rightarrow 0$ or $\Pi_{1} \rightarrow \infty$, no finite limits exist for the function $\Phi$ and also the above exception does not hold good. This case can be said to be lack of similarity of the phenomenon in the parameters $\Pi_{1}$. 
The main challenge in constructing the self-similar variables is that, we need to know the solution of the non-idealized problem a priory to make suitable assumptions of different kinds of self-similarity. To handle such problems, each of the similarity parameters is assumed various kinds of similarity depending on the available experimental data and again comparing the derived relation under each assumption with the experimental or numerical data.

\section{Formulation of fatigue crack propagation model}

The fatigue crack propagation model presented in Ray \& Chandra Kishen (2011) is revisited and modified to consider the effect of varying amplitude loading. The crack growth rate $\frac{d a}{d N}$ in the intermediate amplitude range is observed to be dependent on the following parameters

$$
\frac{d a}{d N}=\Phi\left(\Delta G, G_{f}, \sigma_{t}, R, a, D, \omega, t\right) .
$$

In the above equation, the variables considered are the crack length $(a)$, the loading ratio $(R)$, loading frequency $(\omega)$, time $(t)$, the depth of the beam $(D)$, the tensile strength $\left(\sigma_{t}\right)$ and the size independent fracture energy $\left(G_{f}\right)$. Loading parameter is introduced in the form of change in energy release rate $(\Delta G)$. Considering a state of no explicit time dependence and since $G_{f}, \sigma_{t}$ have independent physical dimensions, dimensional analysis gives

$$
\frac{d a}{d N}=\left(\frac{G_{f}}{\sigma_{t}}\right) \Phi\left(\frac{\Delta G}{G_{f}}, \frac{\sigma_{t}}{G_{f}} a, \frac{\sigma_{t}}{G_{f}} D, R\right),
$$

where the non-dimensional quantities are

$$
\Pi_{1}=\frac{\Delta G}{G_{f}}, \Pi_{2}=\frac{\sigma_{t}}{G_{f}} a, \Pi_{3}=\frac{\sigma_{t}}{G_{f}} D, \Pi_{4}=R .
$$

Now, assuming incomplete self-similarity in the parameters $\Pi_{1}$ and $\Pi_{2}$, it can be written as

$$
\begin{aligned}
\frac{d a}{d N} & =\left(\frac{G_{f}}{\sigma_{t}}\right)\left(\frac{\Delta G}{G_{f}}\right)^{\gamma_{1}}\left(\frac{\sigma_{t}}{G_{f}} a\right)^{\gamma_{2}} \Phi_{2}\left(\Pi_{3}, \Pi_{4}\right) \\
& =G_{f}^{1-\gamma_{1}-\gamma_{2}} \Delta G^{\gamma_{1}} \sigma_{t}^{\gamma_{2}-1} a^{\gamma_{2}} \Phi_{2}\left(\Pi_{3}, \Pi_{4}\right) .
\end{aligned}
$$

Here, the exponents $\gamma_{1}, \gamma_{2}$ and the dimensionless parameter $\Phi_{2}$, cannot be determined from dimensional analysis alone. In this study, these parameters are computed from a best fitting procedure using the experimental results available in the literature through a calibration study.

Further, the above model for constant amplitude loading has been improved to capture the increase in crack propagation rate due to application of varying amplitude loading. From the experimental study (Slowik et al 1996), the increase in crack growth rate due to overload is found to be strongly dependent on process zone ahead of the crack tip. This behaviour suggests that the increase in crack propagation is also influenced by the fracture toughness $G_{f}$. Further, the other parameters such as maximum value of energy release $G_{\max }$ and the crack length $\left(a_{\text {over }}\right)$, which affect the crack propagation significantly need to be taken into consideration. $G_{\max }$ is the loading parameter, computed for the applied loading of higher stress amplitude. $a_{\text {over }}$ can be defined as the crack length until the application of increased stress. $\Delta G_{1}$ is considered to be 
the main crack driving force for the crack propagation. It is defined as the variation in the energy release in the overload cycle and the normal load. It can be expressed as

$$
\Delta G_{1}=\Delta G_{\text {over }}-\Delta G_{\text {normal }} .
$$

On applying the dimensional analysis procedure to the crack growth problem under consideration, the dependencies can be written as

$$
\frac{d a}{d N}=f\left(\Delta G_{1}, G_{f}, G_{\max }, a_{\text {over }}\right)
$$

Considering $G_{f}$ and $a_{\text {over }}$ to have independent physical dimensions and on applying Buckingham $\Pi$ theorem, the number of arguments are reduced by two.

$$
\frac{d a}{d N}=a_{\mathrm{over}}\left(\frac{\Delta G_{1}}{G_{f}}, \frac{G_{\max }}{G_{f}}\right)
$$

where, the non-dimensional quantities are

$$
\Pi_{5}=\frac{\Delta G_{1}}{G_{f}}, \quad \Pi_{6}=\frac{G_{\max }}{G_{f}} .
$$

By analysing the experimental data (Shah 2009), it is confirmed that $\frac{\Delta G_{1}}{G_{f}}$ is a small number, less than 1 . The assumption of complete self-similarity in $\Pi_{5}$ will make the crack growth independent of loading parameter $\Delta G_{1}$, . Hence, incomplete self-similarity in $\Pi_{5}$ is considered and Equation 15 takes the form

$$
\frac{d a}{d N}=a_{\text {over }}\left(\frac{\Delta G_{1}}{G_{f}}\right)^{\beta_{1}} f_{1}\left(\frac{G_{\max }}{G_{f}}\right) .
$$

Now, the crack propagation rate in a structure loaded with varying stress amplitude can be predicted by considering both the Equations 12 and 15. The final form of the fatigue model is given by

$$
\frac{d a}{d N}=\underbrace{\left(\frac{G_{f}}{\sigma_{t}}\right)\left(\frac{\Delta G}{G_{f}}\right)^{\gamma_{1}}\left(\frac{\sigma_{t}}{G_{f}} a\right)^{\gamma_{2}} \Phi_{2}\left(\Pi_{3}, \Pi_{4}\right)}_{\text {Normal load }}+\underbrace{a_{\text {over }}\left(\frac{\Delta G_{1}}{G_{f}}\right)^{\beta_{1}} f_{1}\left(\frac{G_{\max }}{G_{f}}\right)}_{\text {Overload }} .
$$

The coefficients introduced during the dimensional analysis formulation are $\gamma_{1}, \gamma_{2}, \Phi_{2}, \beta_{1}$ and $f_{1}$. It is important to note that, these coefficients remain constant irrespective of the geometry and loading pattern. In the next section, these unknown constants have been computed through a calibration study using available experimental data.

\section{Calibration study}

To calibrate the proposed model, the experimental data of Shah (2009) has been used. Shah (2009), has conducted tests on three-point-bend beams of three geometrically similar sizes. The specimen geometry and material property details are reported in table 1. Sinusoidal loading of varying stress amplitude is applied with a frequency of $1 \mathrm{~Hz}$. The maximum amplitude of the 
Table 1. Geometry and material properties of beam specimens Slowik et al (1996) and Shah (2009).

\begin{tabular}{lccccccc}
\hline $\begin{array}{l}\text { Specimen } \\
\text { designation }\end{array}$ & $\begin{array}{c}\text { Depth } \\
\mathrm{D}(\mathrm{mm})\end{array}$ & $\begin{array}{c}\text { Span } \\
\mathrm{S}(\mathrm{mm})\end{array}$ & $\begin{array}{c}\text { Thickness } \\
\mathrm{B}(\mathrm{mm})\end{array}$ & $\begin{array}{c}\text { Notch size } \\
a(\mathrm{~mm})\end{array}$ & $\begin{array}{c}G_{f} \\
(\mathrm{~N} / \mathrm{mm})\end{array}$ & $\begin{array}{c}E \\
(\mathrm{MPa})\end{array}$ & $\begin{array}{c}\sigma_{t} \\
(\mathrm{MPa})\end{array}$ \\
\hline Beam (small) Shah (2009) & 76 & 190 & 50 & 15.2 & 0.07 & 30000 & 3.9 \\
Beam (medium) Shah (2009) & 152 & 380 & 50 & 30.4 & 0.07 & 30000 & 3.9 \\
Beam (large) Shah (2009) & 304 & 760 & 50 & 60.8 & 0.07 & 30000 & 3.9 \\
$\begin{array}{l}\text { Compact tension (small) } \\
\text { Slowik et al (1996) }\end{array}$ & 300 & - & 100 & 120 & 0.1 & 16000 & 2.03 \\
\hline
\end{tabular}

sinusoidal applied load was increased by $0.5 \mathrm{kN}$ after every 500 cycles as shown in figure 4 . A minimum load of $0.2 \mathrm{kN}$ is applied for all cycles in order to have contact between the loading device and the specimen and also to avoid any impact loading during application of the fatigue load. To calibrate the model, the data and results of medium size specimens are considered and coefficients for the first part (normal load) of the model are determined using the results of a particular applied stress amplitude. Constants are computed through an optimization process using the principle of least squares. The most appropriate values of the exponents $\gamma_{1}$ and $\gamma_{2}$ are 1.96 and 0.0534 , respectively. Further, it is to be noted that, the quantity $\Phi_{2}$ is a function of structural size $\left(\Pi_{3}\right)$. Figure 5 shows the relationship between $\Phi_{2}$ and the nondimensional ratio $\frac{\sigma_{t}}{G_{f}} D\left(\Pi_{3}\right)$ for different structural sizes. The best fit of the above relationship is given by

$$
\Phi=P_{1}\left\{\log \left(\Pi_{3}\right)\right\}^{3}+P_{2}\left\{\log \left(\Pi_{3}\right)\right\}^{2}+P_{3}\left\{\log \left(\Pi_{3}\right)\right\}+P_{4} .
$$

The values of $P_{i}$ are reported to be $-5.68,77.50,-352.32,533.98$, respectively, $i$ ranging from 1 to 4 . The derived expression can be used to compute the values of $\Phi_{2}$ for any size of the specimen and any grade of concrete.

In the next part of the calibration, the exponents involved in the overload function are obtained. This is done by using the experimental data (Shah 2009) for medium-size beam with an intermediate load amplitude other than the first sequence of the constant amplitude loading. Similar methodology, as used to compute the coefficients of the first part of the model, is followed to calibrate the second part of the proposed law. The parameter $\beta_{1}$ is computed to be 1.11 . It is observed that the best results can be captured during the overload acceleration phase when the

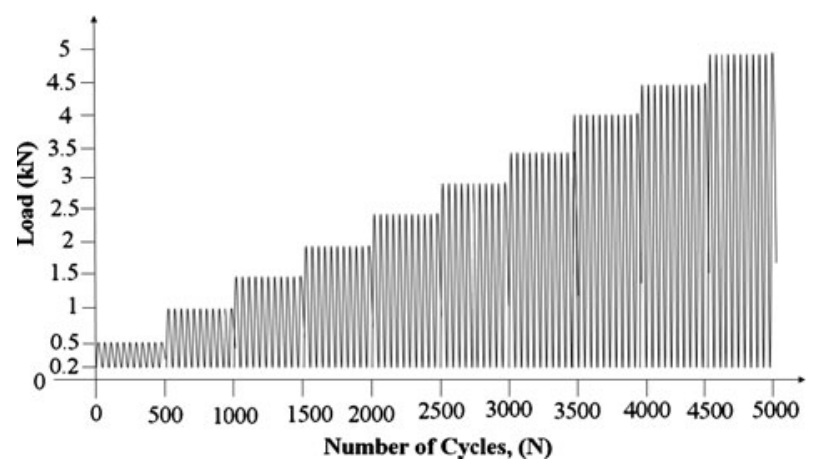

Figure 4. Pattern of fatigue loading. 


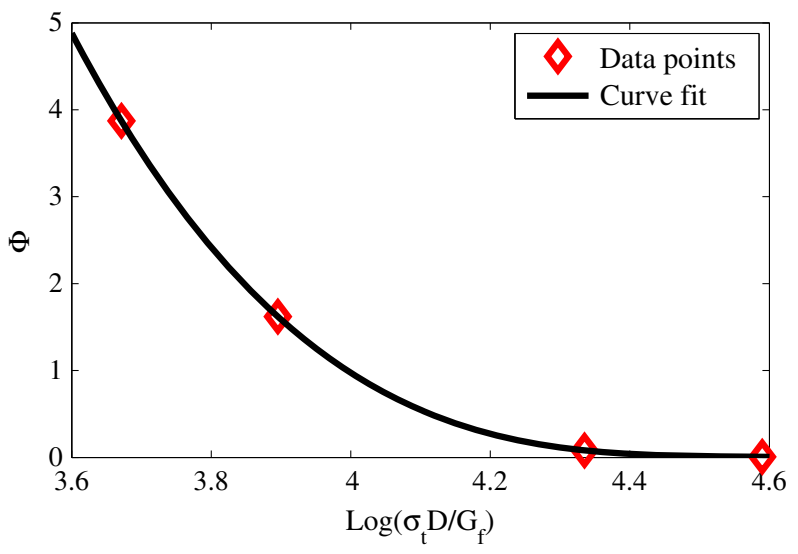

Figure 5. Relationship between $\Phi_{2}$ and the nondimensional parameter.

quantity $\frac{G_{\max }}{G_{f}}$ is expressed in a power law form and is represented as $\left(\frac{G_{\max }}{G_{f}}\right)^{\beta_{2}}, \beta_{2}$ being 1.36. The accuracy of the proposed model is verified using experimental data other than those used to calibrate the model.

In the proposed model described by Equation $18, \Delta G$ is considered only as a loading parameter. Using the concepts of linear elastic fracture mechanics (LEFM), this parameter is related to the stress intensity factor range as

$$
\Delta G=\frac{\Delta K^{2}}{E}
$$

where $E$ is the elastic modulus. According to the concept of LEFM, for compact tension specimen, the equation that relates stress intensity factor and the applied load, specimen geometry and crack size is given by Shah et al (1995)

$$
\Delta K=\frac{\Delta P}{B W^{1 / 2}} f(\alpha),
$$

where $\alpha$ is the relative crack depth equal to $a / D, a$ is the crack length, $D$ is the characteristic dimension of the structure such as depth of the beam, $B$ is the plate thickness, $\Delta P$ is the change in load magnitude during one cycle of loading and $f(\alpha)$ is function of structural geometry and for compact tension specimen is given by

$$
f(\alpha)=\frac{(2+\alpha)\left(0.886+4.64 \alpha-13.22 \alpha^{2}+14.72 \alpha^{3}-5.6 \alpha^{4}\right)}{\left(1-\alpha^{3 / 2}\right)} .
$$

Similarly, for three-point bend beams with the span equal to 2.5 times the depth (Shah et al 1995) is

$$
\begin{gathered}
\Delta K=\frac{3 \Delta P}{2 B W^{1 / 2}} f(\alpha) \\
f(\alpha)=\frac{\left(1-2.5 \alpha+4.49 \alpha^{2}-3.98 \alpha^{3}+1.33 \alpha^{4}\right)}{\left(1-\alpha^{3 / 2}\right)} .
\end{gathered}
$$




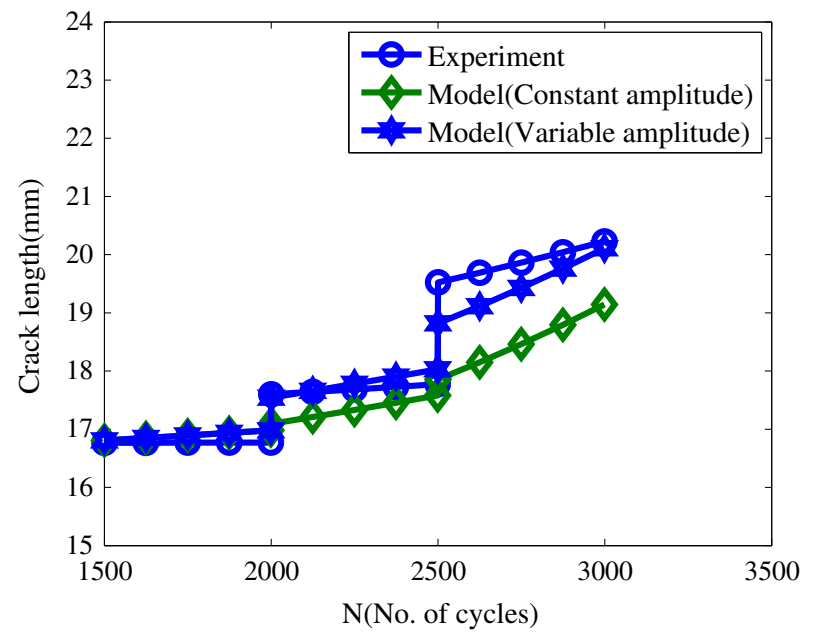

Figure 6. Experimental and computed crack length with the number of load cycles (small size, Shah (2009)).

\section{Validation of the proposed model and discussions}

The proposed fatigue crack propagation model reported in Equation 18 is verified with the experimental data of Shah (2009) and Slowik et al (1996). In the previous section, to compute the model coefficients, the experimental results of medium sized specimen of Shah (2009) has been utilized wherein, among different applied stress ranges on a particular specimen only one stress

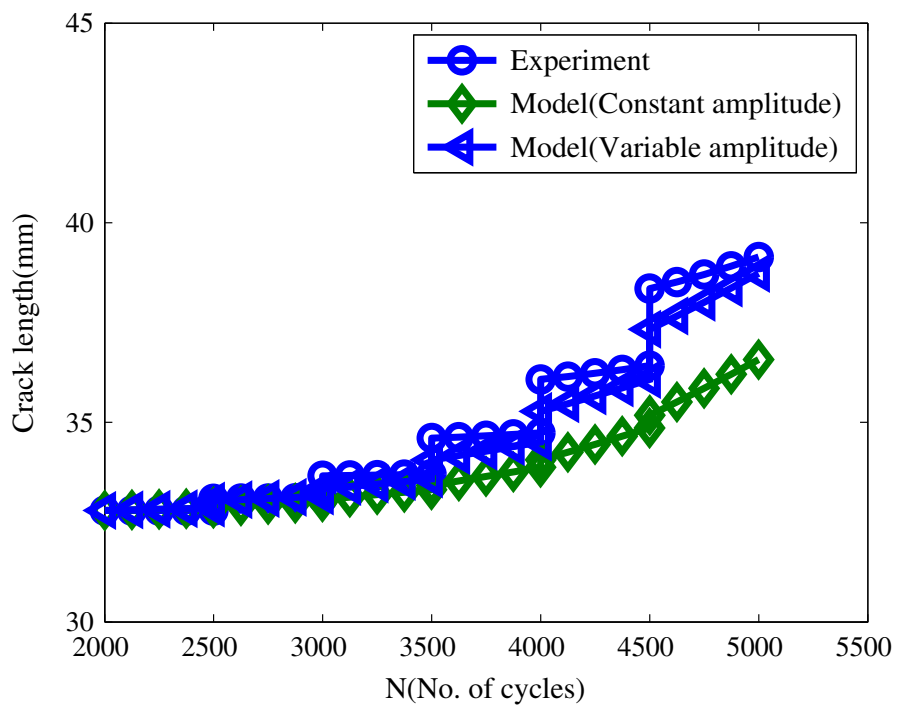

Figure 7. Experimental and computed crack length with the number of load cycles (medium size, Shah (2009)). 


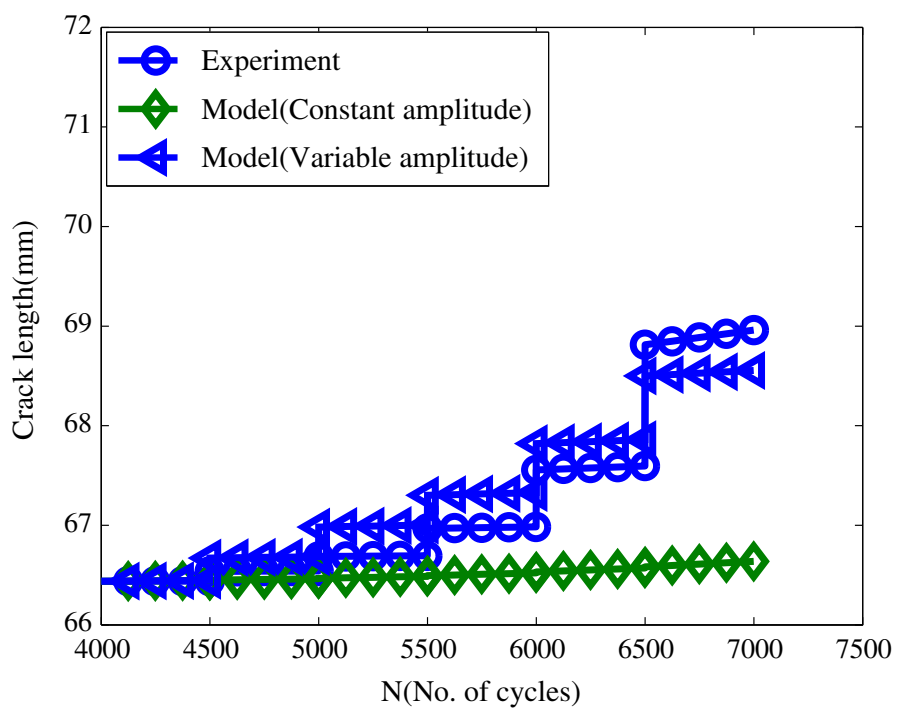

Figure 8. Experimental and computed crack length with the number of load cycles (large size, Shah (2009)).

range has been used for the calibration study. Hence, the applied load ranges of the medium sized specimen other than the one used for the calibration study is utilized to validate the model. The experimental results which fall in the linear range of crack growth curve are considered in this study. On implementing the developed model given in Equation 18, crack growth due to the application of variable amplitude cyclic loading is predicted. Crack length predictions as a function of applied load cycle is plotted for small, medium and large size specimens together with the

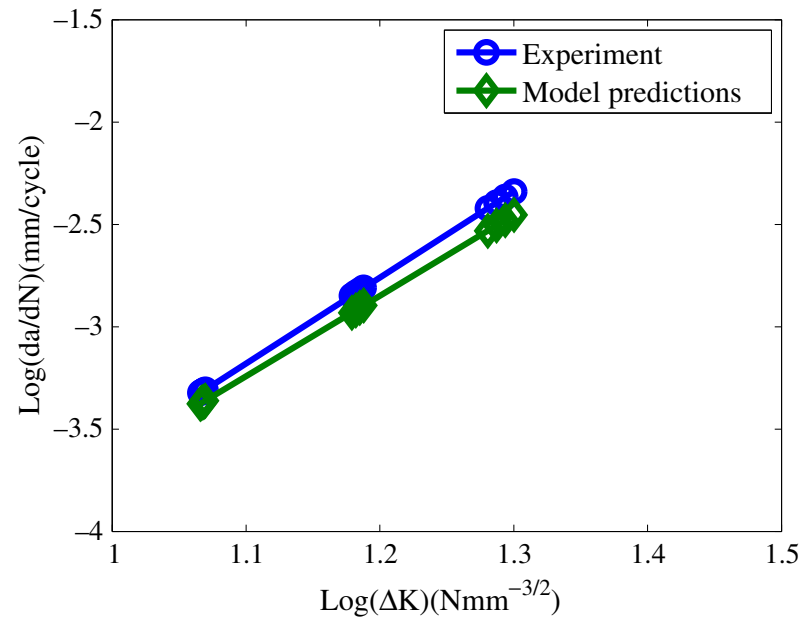

Figure 9. Crack growth rate versus stress intensity factor amplitude (small size, Shah (2009)). 


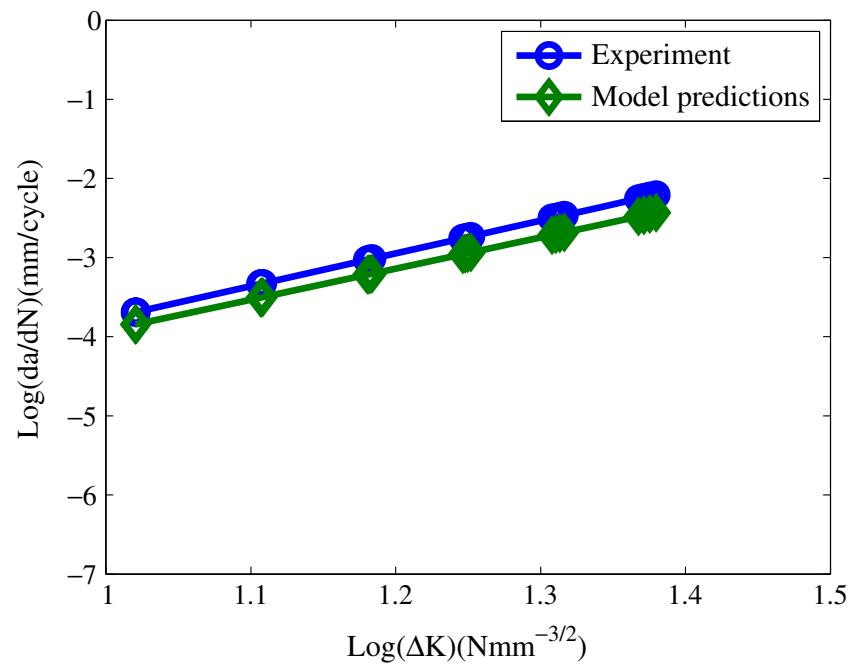

Figure 10. Crack growth rate versus stress intensity factor amplitude (medium size, Shah (2009)).

experimental results in figures 6-8, respectively. In these figures, after every 500 cycles, a sudden increase in crack length is observed due to the applied overloads. Generally, it is observed that damage and stiffness degradation due to application of cyclic loading primarily occur in the fracture process zone and hence can be considered to be more sensitive to fatigue loading than the uncracked portion. In such situations, the overloads in the loading history accelerates the crack growth process and is reflected in figures 6-8. In all the three cases, the proposed model is able to capture the accelerated crack growth on the application of higher amplitude load. Also, the experimental results and model predictions agree well.

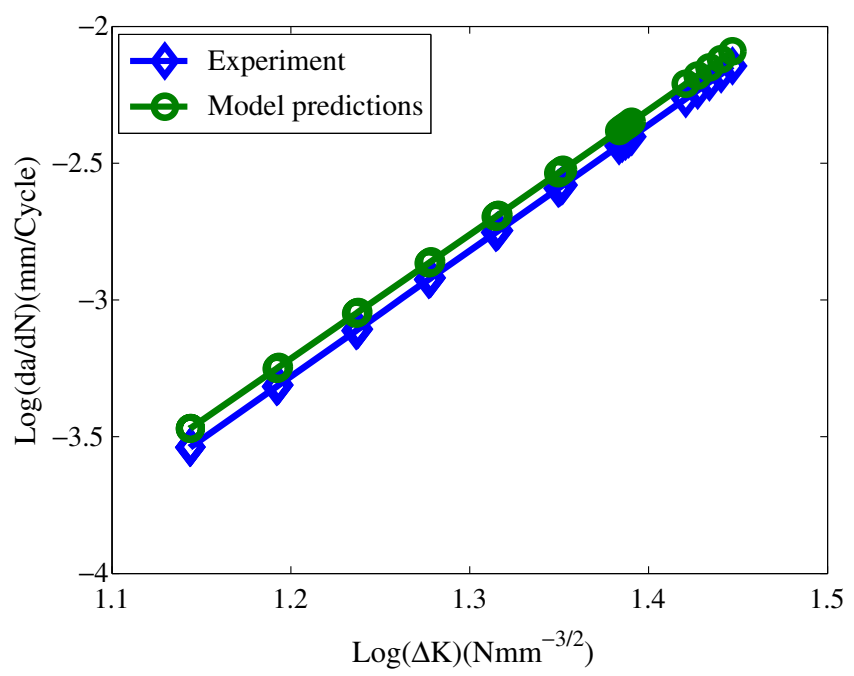

Figure 11. Crack growth rate versus stress intensity factor amplitude (large size, Slowik et al (1996)). 
Table 2. Paris law parameters.

\begin{tabular}{lccccc}
\hline \multirow{2}{*}{ Specimen } & \multicolumn{2}{c}{ Proposed model } & & \multicolumn{2}{c}{ Experiment } \\
\cline { 2 - 3 } \cline { 5 - 6 } & $\log \mathrm{C}$ & & & $\log \mathrm{C}$ & $\mathrm{m}$ \\
\hline Beam (small) & -7.6 & 3.9 & -7.8 & 4.2 \\
Beam (medium) & -7.9 & 3.9 & -7.9 & 4.1 \\
Beam (large) & -8.7 & 4.5 & -8.8 & 4.6 \\
\hline
\end{tabular}

The crack growth rate is computed using the proposed model for small, medium and large specimens and plotted as a function of stress intensity factor range in logarithmic plot as shown in figures 9-11 respectively along with the experimental results. From these curves, the Paris law coefficients $C$ and $m$ are computed and are tabulated in table 2. A good agreement is seen between the proposed model and experimental results. Further, it is observed that the variations in the values of $\log C$ and $m$ for different sizes are not significant. In a study by Bazant \& Xu (1991), they have proposed a size adjusted Paris law by combining size effect law (Bazant \& Planas 1998) into Paris law and this effect has been reflected through minimal variations in $C$ and $m$ values for various structural sizes. A similar observation is made in the present study suggesting that the proposed model is able to capture the size effect and can be employed to predict crack growth in concrete.

In the next part of the validation study, the experimental data of Slowik et al (1996) has been used wherein, the loading pattern was interrupted by spikes. They have conducted testing on compact tension specimens (CT) for both small and large size specimens. The details of dimensions and material properties are reported in table 1 . The proposed model has been implemented to compute crack growth of one specimen from small size. Figure 12 shows the crack length versus number of load cycles for small specimen along with the experimental points and seen to

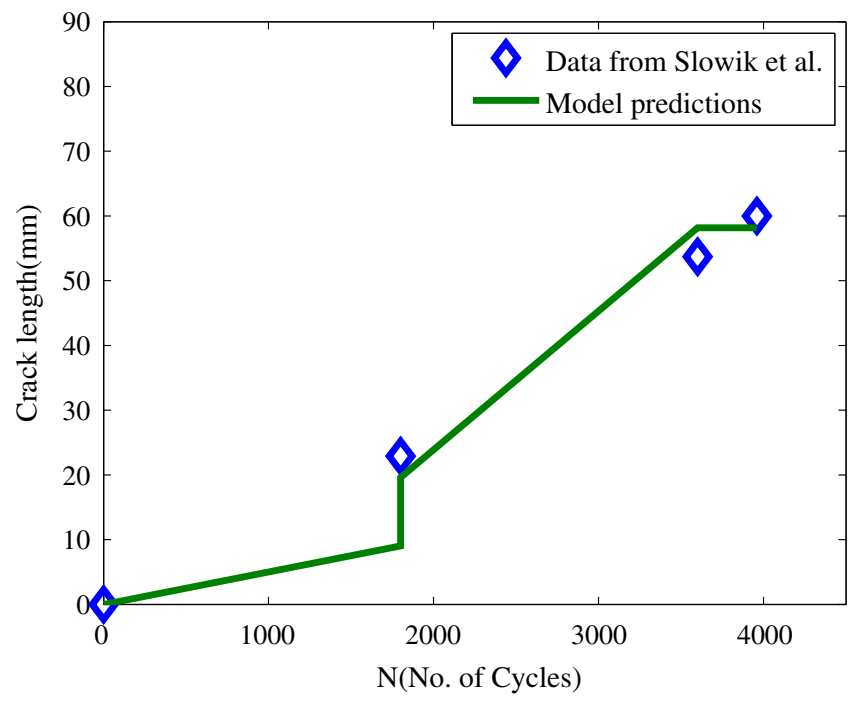

Figure 12. Experimental and computed crack length with the number of load cycles (small size, Slowik et al (1996)). 
match closely. It is observed that the model is able to capture the effect of the spike, indicated by a sudden increase in crack growth at around 1800 load cycle.

\section{A sensitivity study on proposed model}

The model proposed in the previous section to capture the increase in crack length due to the application of overload is influenced by various parameters such as fracture toughness $G_{f}$, maximum value of energy release $\left(G_{\max }\right)$, crack length until the application of increased load $a_{\text {over }}$ and change in energy due to overload $\Delta G_{1}$. Most of the involved parameters are randomly distributed in nature. To understand which of these quantities are more sensitive to the crack growth rate due to overload, a sensitivity study is performed. In this study, the influence in the variation of the maximum number of load cycles $N_{f}$ which corresponds to the crack length at the overload cycle, on the variability of input random quantities is studied. A statistical method is used to compute the sensitivity coefficients by considering the various input parameters as random. The sensitivity coefficient is computed by knowing the coefficient of variation using

$$
p_{i}=100 \frac{v_{y i}^{2}}{v_{y}^{2}}
$$

where $v_{y i}$ is the coefficient of variation of the output quantity keeping the $i$ th input parameter as random and others deterministic; where, $i=1,2, \ldots . N$ and $N$ is the number of input quantities examined in the sensitivity analysis. $v_{y}$ is the coefficient of variation of the output quantity, considering all the input quantities as random ones. The concept is based on the assumption that higher value of sensitive coefficient $p_{i}$ indicates higher degree of correlation and therefore higher influence of input variable on the output (Sain \& Chandra Kishen 2008).

The number of load cycles $N_{f}$ required to achieve the sudden increase in crack length of concrete member is computed using the proposed fatigue law (Equation 18) as

$$
\int_{0}^{N_{f}} d N=\int_{a_{0}}^{a} \frac{d a}{\left(\frac{G_{f}}{\sigma_{t}}\right)\left(\frac{\Delta G}{G_{f}}\right)^{\gamma_{1}}\left(\frac{\sigma_{t}}{G_{f}} a\right)^{\gamma_{2}} \Phi_{2}\left(\Pi_{3}, \Pi_{4}\right)+a_{\mathrm{over}}\left(\frac{\Delta G_{1}}{G_{f}}\right)^{\beta_{1}} f_{1}\left(\frac{G_{\max }}{G_{f}}\right)}
$$

or

$$
N_{f}=\int_{a_{0}}^{a} \frac{d a}{\left(\frac{G_{f}}{\sigma_{t}}\right)\left(\frac{\Delta G}{G_{f}}\right)^{\gamma_{1}}\left(\frac{\sigma_{t}}{G_{f}} a\right)^{\gamma_{2}} \Phi_{2}\left(\Pi_{3}, \Pi_{4}\right)+a_{\mathrm{over}}\left(\frac{\Delta G_{1}}{G_{f}}\right)^{\beta_{1}} f_{1}\left(\frac{G_{\max }}{G_{f}}\right)},
$$

where $a_{0}$ and $a$ are the initial and crack length reached after application of higher amplitude loading respectively and $\Phi_{2}$ is defined by the Equation 19 .

The Latin Hypercube Sampling (LHS) method is used to generate random distribution of the random variable in the numerical simulation by knowing the type of distribution, the mean and standard deviation. Using these randomly obtained samples the coefficient of variation is computed. The sensitivity analysis is carried out on the large sized beam specimen of Shah (2009). The sensitivity coefficients are computed considering the input parameters $G_{f}, G_{\max }$, 


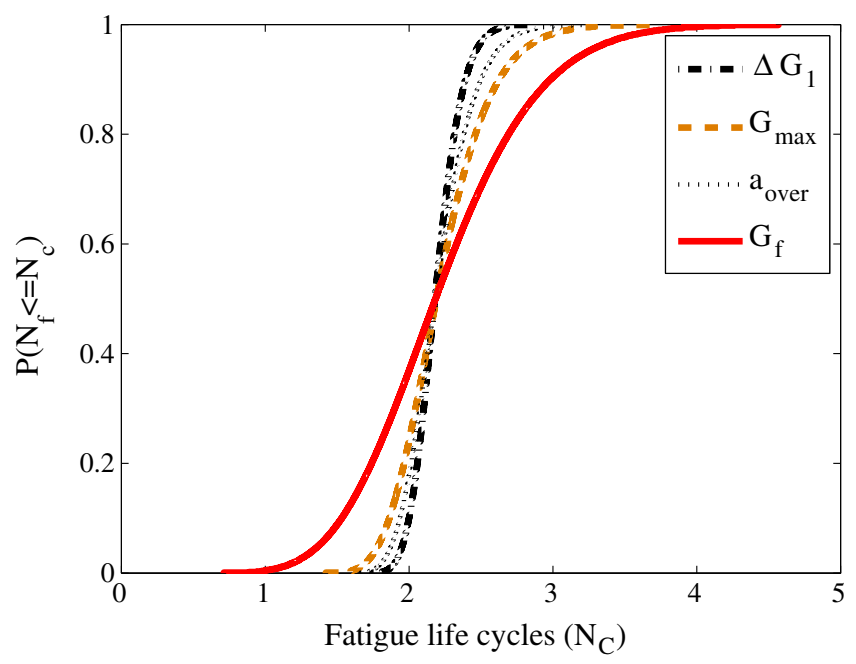

Figure 13. Distribution curves of fatigue life $N_{f}$ for different random variables.

$a_{\text {over }}$ and $\Delta G_{1}$ as random variables. The statistical distributions (Kala 2006) for different input random variables are given in table 3 together with the coefficient of variations. The quantity with maximum coefficient of variation is considered to be the most sensitive one. It is observed from table 3 that fracture toughness is the most sensitive parameter followed by $G_{\max }$ and $a_{\text {over }}$. It is to be noted that $\Delta G_{1}$ possess a least sensitivity coefficient value compared to the other parameters. In case of fatigue loading, FPZ is considered to be responsible for the variation of material properties than the uncracked ligament. Introduction of higher amplitude cyclic loading is likely to accelerate the crack growth process (Slowik et al 1996) as FPZ is more sensitive than the uncracked portion. Hence, fracture toughness $\left(G_{f}\right)$ which is related to process zone is also observed to have greater sensitivity towards the application of overload.

Further, to understand the correctness in the computation of sensitivity coefficients of the different influencing parameters, the results of the sensitivity analysis are plotted in terms of probability distribution function. Figure 13 shows the computed probability distribution functions, wherein the actual number of load cycles $N_{c}$ is plotted against the chance that the computed life $N_{f}$ is less than a specific $N_{c}$ value. The $N_{f}$ values are computed through Monte-Carlo simulation considering one parameter as random at a time and keeping other parameters as deterministic. If the probability distribution function depicts a steeper (more or less vertical) curve, then it implies that the parameter is not much sensitive and thus can be considered as deterministic. The results of distribution curve also show a similar trend to the ones observed in table 3.

Table 3. Statistical parameters used in the sensitivity studies.

\begin{tabular}{lcccc}
\hline Variables & Distribution & Mean & $\begin{array}{c}\text { Standard } \\
\text { deviation }\end{array}$ & $\begin{array}{c}\text { Coefficient of } \\
\text { variation }\left(p_{i}\right) \%\end{array}$ \\
\hline$G_{f}(\mathrm{~N} / \mathrm{mm})$ & Normal & 0.07 & 0.007 & 60.42 \\
$G_{\max }(\mathrm{N} / \mathrm{mm})$ & Normal & 0.0088 & 0.00088 & 17.27 \\
$a_{\text {over }}(\mathrm{mm})$ & Normal & 66.45 & 6.45 & 9.55 \\
$\Delta G_{I}(\mathrm{~N} / \mathrm{mm})$ & Normal & 0.0016 & 0.0001 & 4.54 \\
\hline
\end{tabular}




\section{Conclusions}

A crack propagation model has been proposed for plain concrete to capture the effect of overload. The fatigue law consists of two parts, wherein the first part is used to predict crack growth rate due to constant amplitude load cycles and the second one captures the increase in crack growth due to overload. The proposed model is developed by utilizing the fundamental principles of dimensional analysis and self-similarity, thereby overcoming the empirical nature of the analytical models. The model, accounts for many crack growth influencing parameters such as the tensile strength, fracture toughness, loading ratio, initial crack length, overload effect and structural size. Further, the proposed model has been validated with available experimental data of three-point bend beams and compact tension specimens and is found to be in good agreement with experimental data. Through a sensitivity analysis, the fracture toughness is found to be the most dominant parameter in accelerating the crack growth due to application of an overload.

\section{Nomenclature}

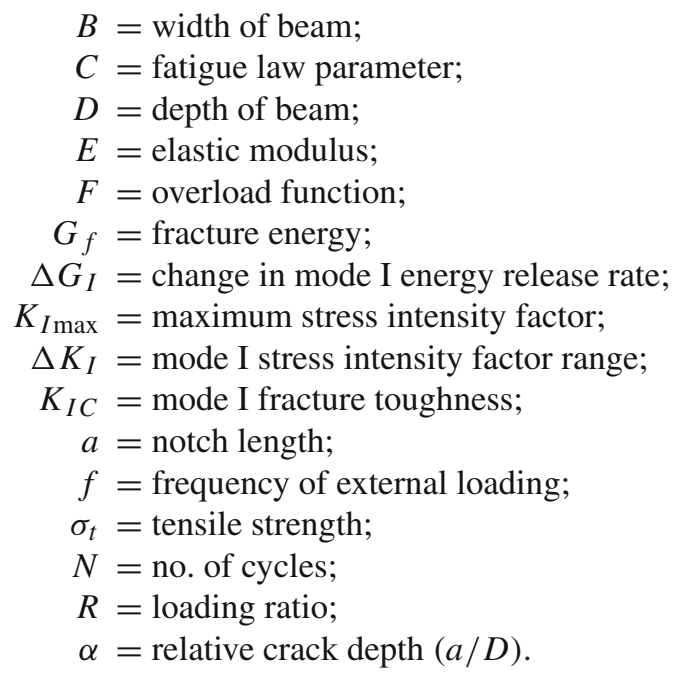

\section{References}

Barenblatt G I 1996 Scaling, self-similarity and intermediate asymptotic. (New York: Cambridge University Press)

Barenblatt G I 2004 Scaling. (New York: Cambridge University Press)

Bazant Z P and Xu K 1991 Size effct in fatigue fracture of concrete. ACI Mater. J. 88(4): 390-399

Bazant Z P and Planas J 1998 Fracture and size effect in concrete and other quasi brittle materials. (New York: CRC Press)

Carpinteri A and Spagnoli A 2004 A fractal analysis of size effect on fatigue crack growth. Int. J. Fatigue 26: $125-133$

Carpinteri A and Paggi M 2007 Self-similarity and crack growth instability in the correlation between the Paris constants. Eng. Fracture Mech. 74:1041-1053

Carpinteri A and Paggi M 2009 A unified interpretation of the power laws in fatigue and the analytical correlations between cyclic properties of engineering materials. Int. J. Fatigue 31: 1524-1531 
Carpinteri A, Spagnoli A and Vantadori S 2009 Size effect in S-N curves: A fractal approach to finite-life fatigue strength. Int. J. Fatigue 31(5): 927-933

Ciavarella M, Paggi M and Carpinteri A 2008 One, no one and one hundred thousand crack propagation laws: A generalized Barenblatt and Botvina dimensional analysis approach to fatigue crack growth. J. Mechanics and Physics of Solids 56: 3416-3432

Cornelissen H A W and Reinhardt H W 1984 Uniaxial tensile fatigue failure of concrete under constant amplitude and program loading. Mag. Concr. Res. 36(129): 216-226

Hilsdorf H K, Kesler C E 1964 Fatigue strength of concrete under varying flexural stresses. ACI J. 63(10): 1059-1076

Holmen J O 1979 Fatigue of concrete by constant and variable amplitude loading. Ph.D. thesis, Norwegian Institute of Technology, University of Trondheim

Hordijk D A and Reinhardt H A 1989 Influence of load history on mode I fracture of concrete. In H. Mihashi et al (eds.) Fracture toughness and fracture energy: Test methods for concrete and rock. Balkema, Rotterdam, pp. 35-46

Kala Z 2006 Sensitivity analysis of fatigue behaviour of steel structure under in-plane bending. Nonlinear Anal. Model. Control 11(1): 33-45

Oh B H 1993 Cumulative damage theory of concrete under variable amplitude fatigue loading. ACI Materials J. 88(1): 41-48

Paggi M 2009 A dimensional analysis approach to fatigue in quasi-brittle materials. Frattura ed Integrita Strutturale 10: 43-55

Ray S and Chandra Kishen J M 2010 Fatigue crack propagation model for plain concrete - An analogy with population growth. Eng. Fracture Mech. 77: 3418-3433

Ray S and Chandra Kishen J M 2011 Fatigue crack propagation model and size effect in concrete using dimensional analysis. Mech. Mater. 43(129): 75-86

Ruzicka M C 2008 On dimensionless numbers. Chem. Eng. Res. and Design 86: 835-868

Sain T and Chandra Kishen J M 2007 Prediction of fatigue strength in plain and reinforced concrete beams. ACI Mater. J. 104 (5): 621-628

Sain T and Chandra Kishen J M 2008 Probabilistic assessment of fatigue crack growth in concrete. Int. J. Fatigue 30: 2156-2164

Shah S G 2009 Fracture and fatigue behavior of concrete-concrete interfaces using acoustic emission, digital image correlation and micro-indentation techniques. Ph.D. thesis, Department of Civil Engineering, Indian Institute of Science, Bangalore, India

Shah S P, Swart S E and Ouyang C 1995 Fracture mechanics of concrete: Applications to concrete, rock and other quasi-brittle materials. (New York: John Wiley and Sons)

Slowik V, Plizzari G A and Saouma V E 1996 Fracture of concrete under variable amplitude fatigue loading. ACI Mater. J. 93(3): 272-283

Spagnoli A 2004 Self-similarity and fractals in the Paris range of fatigue crack growth. Mech. Mater. 37: $519-529$ 